\title{
Analog-digital hybrid impression technique in an elderly patient: a case report
}

\author{
Efstratios Papazoglou ${ }^{1}$ and Constantinos Charalambous ${ }^{1}$ \\ ${ }^{1}$ Affiliation not available
}

January 7, 2021

\begin{abstract}
The hybrid impression technique consists of an initial alginate impression that provides a pre-operative cast upon which a diagnostic wax-up and a silicone index impression are made. This work is digitized; thus, the altered final digital impression is limited to absolute minimum time, effort and ensures comfort for the patient.
\end{abstract}

\section{Introduction}

Implant therapy in elderly patients is not considered a contradiction as long as is permitted by the general health of the individual, contemplating that multimorbidity and polypharmacy occur in greater incidence than in younger people. ${ }^{1}$ Studies have showed no significant difference of crestal bone loss over a span of 17 years between older and younger adults. ${ }^{2}$ At the same time, old age does not seem to give rise to significantly different survival rates of implants and fixed implant supported prostheses, at least on edentulous patients. ${ }^{3,4}$ Focus should be given on the expectations and willingness of the patient to undergo surgical procedures as well as the ability in handling and caring for their implant supported prosthesis. ${ }^{5,6}$

Additionally, elderly patients may be less able to cope well with prolonged and complex surgical procedures and multiple appointments. Computer designed surgical templates assist the operator to complete the implant placement in reduced operating time as well as reduced postoperative discomfort, aspects especially important when treating elderly patients. ${ }^{7,8}$ Digitally designed surgical guides provide during surgery, implant positions that are prosthetically driven according to the existing 3D osseous topography as projected in the CBCT. ${ }^{9}$ Such an approach might also reduce the necessity for additional grafting procedures.

The aim of this article was to describe step by step a hybrid impression technique where the study cast, produced by the initial alginate impression, was digitized and locally altered by a final intraoral digital impression at the day of implant placement. The main objective while treating this case was to decrease the duration and the number of the appointments without compromising the quality of the final result. Based on this, a combination of conventional and digital impressions was used to exploit the fact the that the patient presented with the fixed prosthesis detached. Deviation from the fully digital protocol, using analog alginate initial impressions, possessed advantages both on template designing, as well as on saving time during the final corrected digital impression, which took place at the same session with implant placement and when the elderly patient was mostly vulnerable to fatigue.

\section{CASE HISTORY / examination}

An 86-year-old woman, unable to commute alone, presented escorted by her daughter, with a detached mandibular anterior FPD, previously supported on the mandibular left central incisor and right canine. Clinical examination revealed that both abutment teeth were decayed and fractured while the root of the left incisor was completely covered by soft tissues (Fig. 1). They both had bad prognosis. Cone beam 
computed tomography (CBCT) revealed adequate amount of alveolar bone at the positions of left central and right lateral incisors as sufficient for implant placement without a need for regenerative procedures. Maximum intercuspation was stable and reproducible with no premature contacts and mandible slide. The patient declared that she did not want to receive a removable prosthesis nor prepare the existing teeth for a Fixed Partial Denture (FPD). Therefore, the treatment plan involved an implant supported FPD

\section{TREATMENT}

At the first appointment alginate impressions (Hydrogum 5; Zhermack) of maxillary and mandibular arches were made for treatment planning. Diagnostic casts were fabricated using high strength, low expansion and high accuracy Type IV dental stone (Elite Master; Zhermack). After 30 min setting time the edentulous mandibular cast was scanned using an intraoral scanner (Medit i500; Medit Corp) followed by articulation of both casts with the relevant member of a gypsum-free articulator (MagicArt-2; MagicArt) and articulated at maximum intercuspation by tightening the grips with an Allen driver. ${ }^{10}$ The detached FDP was secured in the correct position on the mandibular cast using sticky wax. Corrections were made with carving wax. The waxed up analog mandibular cast was digitized using the same intraoral scanner (Fig. 2 A, B). Both mandibular scans were exported in STL (Standard Tessellation Language) format and superimposed on the CBCT data in dicom format, using an implant planning software (BlueSkyPlan; BlueSkyBio).

The positions of left central and right lateral incisors were selected for placement of 2 internal hexagon implants of $13 \mathrm{~mm}$ in length a narrow for the left central incisor and a standard diameter for the right lateral incisor (Paltop Keystone). The position of right canine was not selected for implant placement due to an apical fenestration of the buccal cortical plate. The dentate stl cast was used for the correct position and angulation of implants (Fig. $3 \mathrm{~A}, \mathrm{~B}, \mathrm{C}, \mathrm{D}$ ). The pilot surgical guide was designed with the same software on the edentulous stl cast and the surgical guide stl file was extracted. It was 3D printed (Max UV 385 3D printer; Asiga) in 45 minutes using a transparent resinous material (Freeprint splint 2.0; Detax). Post processing consisted of ultrasonic cleaning in isopropyl alcohol and ultraviolet polymerization for 20 minutes (Asiga Flash; Asiga). The supportive pins were removed and the guide was finished and polished (Fig. 4).

Implant surgery was scheduled for the second appointment. The patient was under anticoagulation therapy with a selective direct inhibitor of factor Xa (Xarelto [Rivaroxaban]; Bayer Pharma) and discontinuation of the treatment 2 days prior the scheduled surgery was required. Two implants were placed successfully, using the 3D printed pilot guide at the preplanned positions, without the need of any grafting procedure. Primary stability $(>35 \mathrm{Ncm})$ was achieved on both implants, thus, healing abutments were placed right away for a single stage approach. The flap was secured using 5.0 monoclonal absorbable sutures (Monofast; Medipac).

Following suturing, at the same session, the healing abutments were removed and appropriate diameter scan abutments (Standard platform and Narrow platform; Paltop Keystone) were connected to the implants (Fig. 5). A digital altered final impression was made using the intraoral scanner in high resolution mode (Fig. 6 A, B). The intraoral scanning was limited on the area of surgery plus 1 adjacent tooth on each side since the rest of the mandibular arch was already available using the extraoral scanning of the initial study casts. The intraoral post-surgery scanning time was about 30 seconds. By utilizing a digital intraoral local scan, technicalities of the conventional final impression like suture protection with an apron and undercut blocking were avoided. Maximum intercuspation lateral records were taken on the articulated casts.

Healing period was set to 8 weeks. On the third appointment a 3D printed FPD (Resin, DentaModel; Asiga) clinical evaluation was performed, on 2 Ti-base abutments (Paltop Keystone), the narrow platform without and the standard platform with hexagon engagement. The fit, , contact points, emergence profile and occlusion of the prosthesis were inspected (Fig. 7). Minimal widening of the interdental embrasures and occlusal adjustments were deemed necessary. These changes that were incorporated into the final design of a monolithic zirconia FPD.

Three days later, at the fourth appointment an implant supported, screw and cement retained monolithic zirconia FPD (High Translucency, Katana; Kuraray) was delivered successfully to the patient. ${ }^{11,12}$ Final torque of $30 \mathrm{Ncm}$ and $35 \mathrm{Ncm}$ was applied for the narrow and standard platform respectively, following 
manufacture's recommendations. Access holes were restored using Teflon tape (Polytetrafluoroethylene, PTFE) and 2 increments of flowable resin, $1.5 \mathrm{~mm}$ each (Masking liner and G-ænial Universal Flo; GC) (Fig. 8 A, B, C). Patient was instructed on the proper use of interproximal brushes and was scheduled on a 6-month recall.

\section{Discussion}

The treatment workflow selected for the treatment of this geriatric patient was neither the conventional analog, neither the contemporary full digital approach. Conventional initial impression with alginate was made, mainly because the detached FDP was readily available. This allowed for an easy wax up needed for the prosthetically driven implant placement. In addition, alginate as first impression is a fast and comfortable procedure. Most studies that compare the analog and digital impressions, do favor the later. ${ }^{13-15}$ This is probably true for final impression materials like polyether or polyvinyl siloxane, which require longer setting time, they do not taste good and are stiff and more difficult to remove from the mouth, rather than alginate impression. The extraoral digitalization of the diagnostic casts allowed for a simplified and quicker intraoral scanning process limited to the implant site. Furthermore, final digital impression was taken at the same session as implant placement without causing further discomfort to the elderly patient or need for an additional impression appointment.

There is no previous report of such analog-digital impression technique in which the original alginate analog impression is digitized and then a part of it is cut and altered by intraoral scanning in high definition mode of the area of interest. Any inaccuracy of the original alginate impression was nullified by the final intraoral digital impression which included the edentulous area of interest and the proximal teeth. Additionally, any occlusal discrepancies due to the use of the gypsum-free articulator were corrected at the phase of 3D-printed resin FPD clinical evaluation.

This technique can also be used in cases where there is a need for a localized diagnostic wax up and the only available equipment in the dental office, for a full digital work flow is an intraoral scanner. Initial alginate impressions are made, and poured. Meanwhile, the tooth or teeth of interest are prepared. After stone setting the casts are removed from the impressions, trimmed and mounted on a gypsum-free articulator, followed by a quick diagnostic wax up. The advantage of having an analog cast is threefold. Firstly, a silicone matrix can be fabricated and a provisional restoration can be fabricated intraorally in the same appointment. Secondly, the analog cast is extraorally digitized and is used for the localized corrected digital impression. Lastly the digitalized waxed up cast communicates to the dental technician the desired shape of the final restoration.

In an era where a small percentage of dental offices owe a digital scanner ${ }^{16}$ and fewer have a 3D printer, a design software, a CAD-CAM milling unit accompanied with a staining and glazing furnace, all that is needed for a full in-house digital workflow, modified analog-digital techniques bridging the available analog and digital technologies can help us offer a lot more to our patients.

\section{Author contributions}

SP performed the diagnosis, the treatment planning, the treatment as well as the writing of the manuscript. $\mathrm{CC}$ executed the digital design of the surgical guide, the writing of the manuscript and the management of the photographic material.

\section{References}

1. de Baat, C. Success of dental implants in elderly people-a literature review. 2000. Gerodontology 17:45-48.

2. Bryant, S.R., Zarb, G.A. 2003. Crestal bone loss proximal to oral implants in older and younger adults. J Prosthet Dent 89:589-597.

3. Hoeksema, A.R., Visser, A., Raghoebar, G.M., Vissink, A., Meijer, H.J. 2016. Influence of Age on Clinical Performance of Mandibular Two-Implant Overdentures: A 10-Year Prospective Comparative Study. Clin Implant Dent Relat Res 18:745-751. 
4. Engfors, I., Ortorp, A., Jemt, T. 2004. Fixed implant-supported prostheses in elderly patients: a 5-year retrospective study of 133 edentulous patients older than 79 years. Clin Implant Dent Relat Res 6:190-8.

5. Visser, A., de Baat, C., Hoeksema, A.R., Vissink, A. 2011. Oral implants in dependent elderly persons: blessing or burden? Gerodontology 28:76-80.

6. Bergendal, B., Anderson, J.D., Müller, F., Jokstad, A. 2008. Osseointegration and dental implants. $1^{\text {st }}$ ed Wiley-Blackwell 43-62p.

7. Youk, S.Y., Lee, J.H., Park, J.M., et al. 2014. A survey of the satisfaction of patients who have undergone implant surgery with and without employing a computer-guided implant surgical template. J Adv Prosthodont 6:395-405.

8. Hämmerle, C.H., Stone, P., Jung, R.E., Kapos, T., Brodala, N. 2009. Consensus statements and recommended clinical procedures regarding computer-assisted implant dentistry. Int J Oral Maxillofac Implants 24 Suppl:126-131.

9. Tatakis, D.N., Chien, H.H., Parashis, A.O. 2019. Guided implant surgery risks and their prevention. Periodontol 2000 8:194-208.

10. Papazoglou, E., Diamantopoulou, S. 2015. The Modified Semidirect Onlay Technique With Articulated Elastic Model. Eur J Prosthodont Restor Dent 23:207-212.

11. McGlumphy, E.A., Papazoglou, E., Riley, R.L. 1992. The combination implant crown: a cement- and screw-retained restoration. Compendium 13:34-38.

12. Proussaefs, P., AlHelal, A. 2018. The combination prosthesis: A digitally designed retrievable cementand screw-retained implant-supported prosthesis. J Prosthet Dent. 119:535-539.

13. Yuzbasioglu, E., Kurt, H., Turunc, R., Bili,r H. 2014. Comparison of digital and conventional impression techniques: evaluation of patients' perception, treatment comfort, effectiveness and clinical outcomes. BMC Oral Health 14:10.

14. Lee, S.J., Macarthur, R.X. 4th, Gallucci, G.O. 2013. An evaluation of student and clinician perception of digital and conventional implant impressions. J Prosthet Dent 110:420-3.

15. Gjelvold, B., Chrcanovic, B.R., Korduner, E.K., Collin-Bagewitz, I., Kisch, J. 2016. Intraoral Digital Impression Technique Compared to Conventional Impression Technique. A Randomized Clinical Trial. J Prosthodont 25:282-287.

16. Zimmermann, M., Mehl, A., Mörmann, W.H, Reich S. 2015. Intraoral scanning systems - a current overview. Int J Comput Dent 18:101-129.

\section{Figure LEGENTS}

Fig 1. Pretreatment clinical situation, occlusal view.

Fig 2. Screenshots of digitized casts. A. Edentulous with FDP detached. B. Waxed up with FDP reattached

Fig 3. CBCT sagittal views of possible positions for implant placement. Green line delineates the edentulous cast, blue line the digitized model with the FPD reattached. A. Left central incisor. B. Right central incisor. C. Right lateral incisor. D. Right canine with the apical fenestration of the buccal cortical plate.

Fig 4. 3D-printed surgical guide with $2 \mathrm{~mm}$ pilot sleeves in place.

Fig 5. Immediate postoperative intraoral view with scan posts.

Fig 6. Screenshots of analog-digital altered final impression. The display mode was set on Color, denoting actual color of impressed objects. Intraorally scanned anterior area is obvious, in contrast to the extraoral digital scanning of initial stone cast. A. Facial view, B. Occlusal view 
Fig 7. 3D-printed clinical evaluation prosthesis on 2 Ti-base abutments.

Fig 8. Final screw/ cement implant supported FDP of monolithic zirconia. A. Ti-base with hexagon engagement for standard platform (right lateral incisor) and respective Ti-base without hexagon engagement for narrow platform (left central incisor). B. FDP on the 3D-printed cast and access holes at correct lingual positions. C. Final clinical result. Intraoral lingual view of prosthesis with access holes restored.
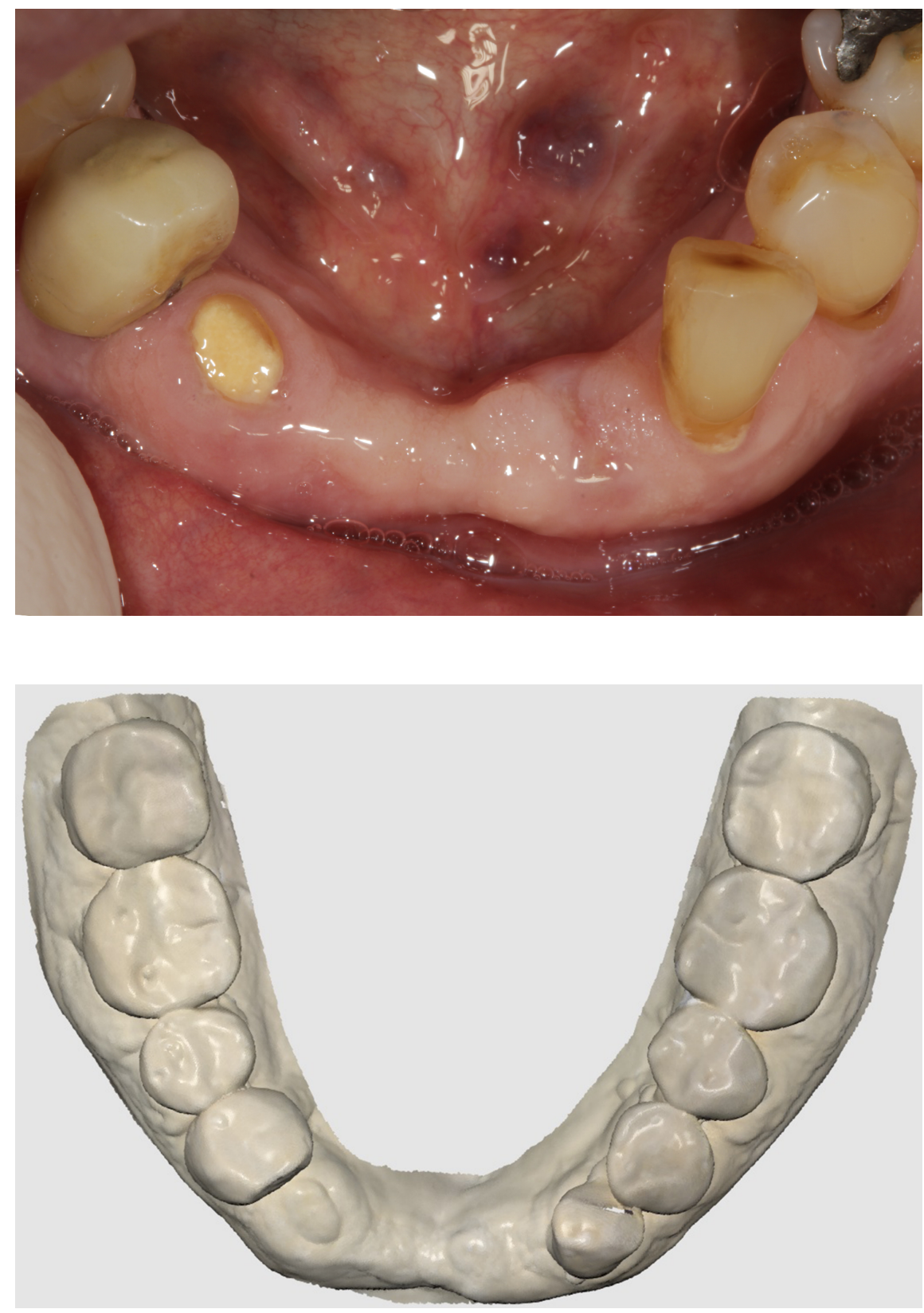

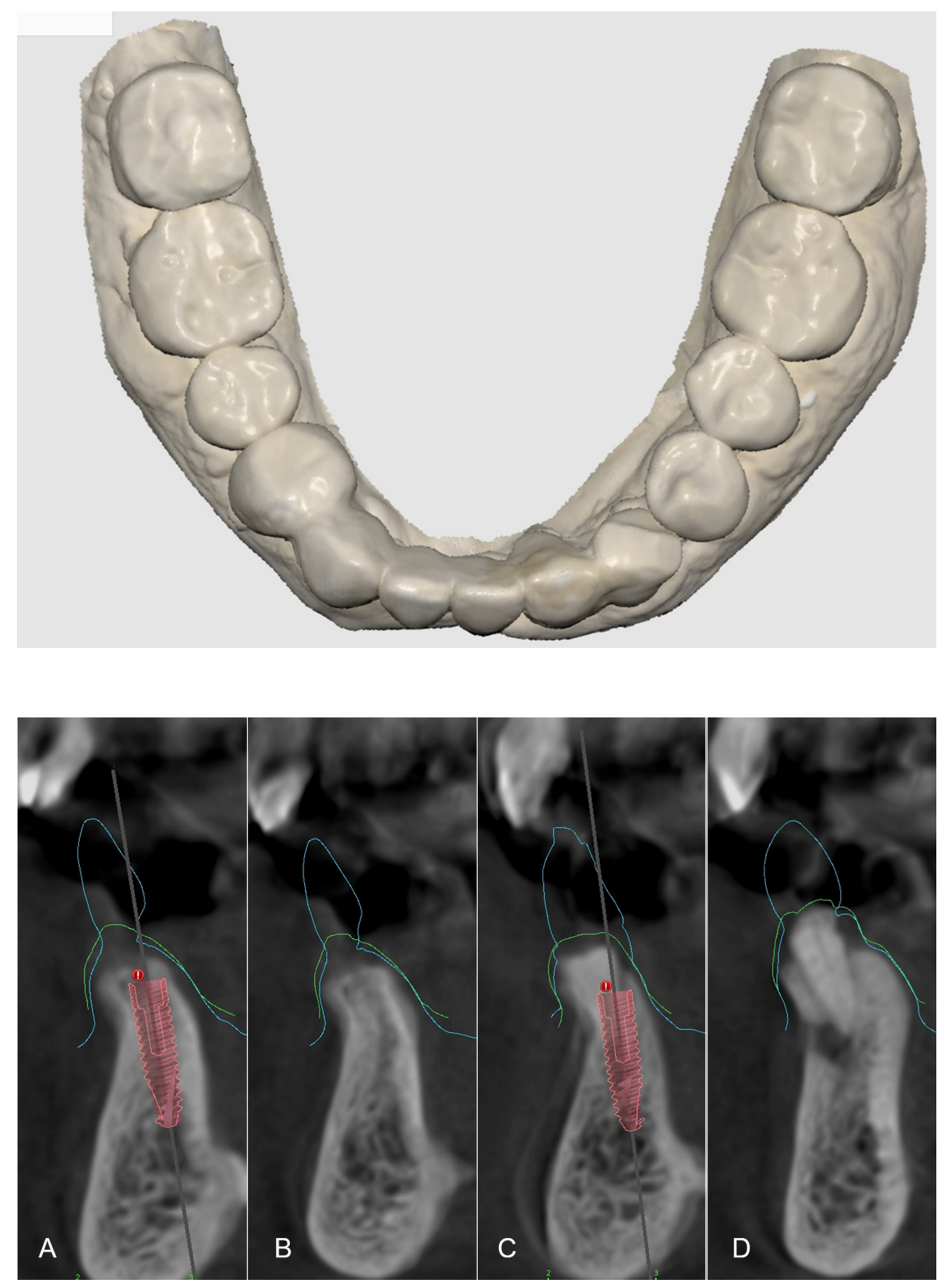

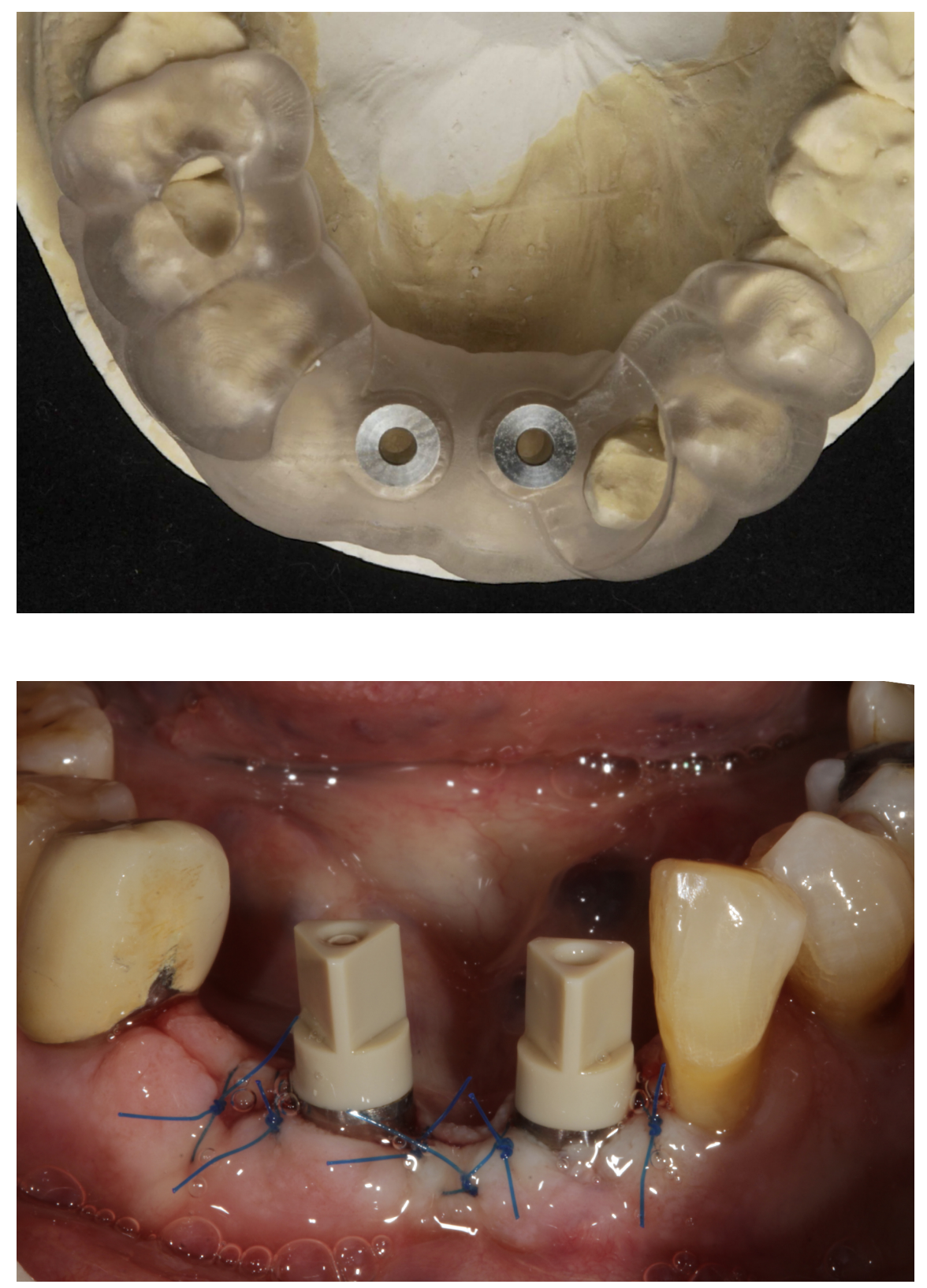

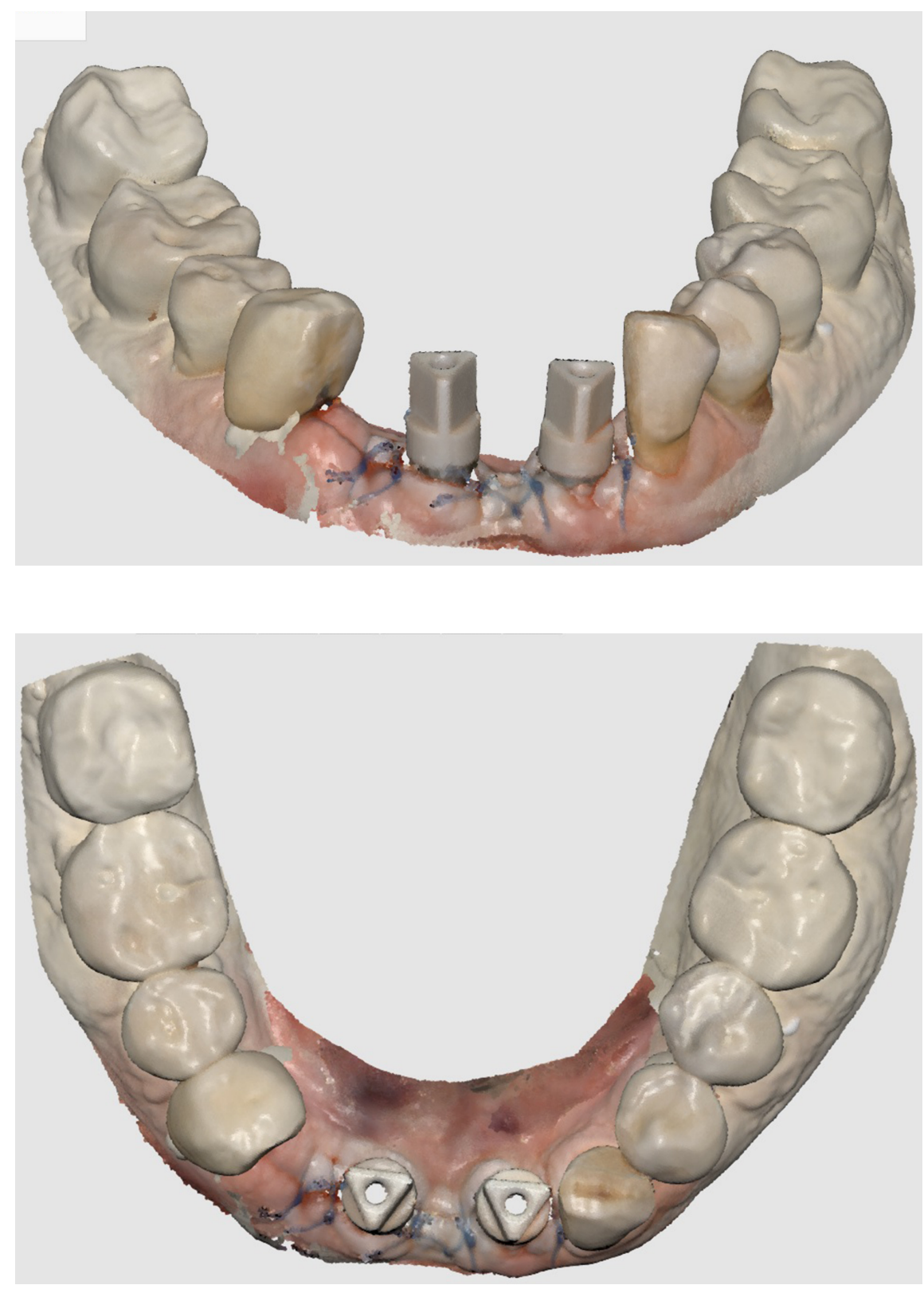

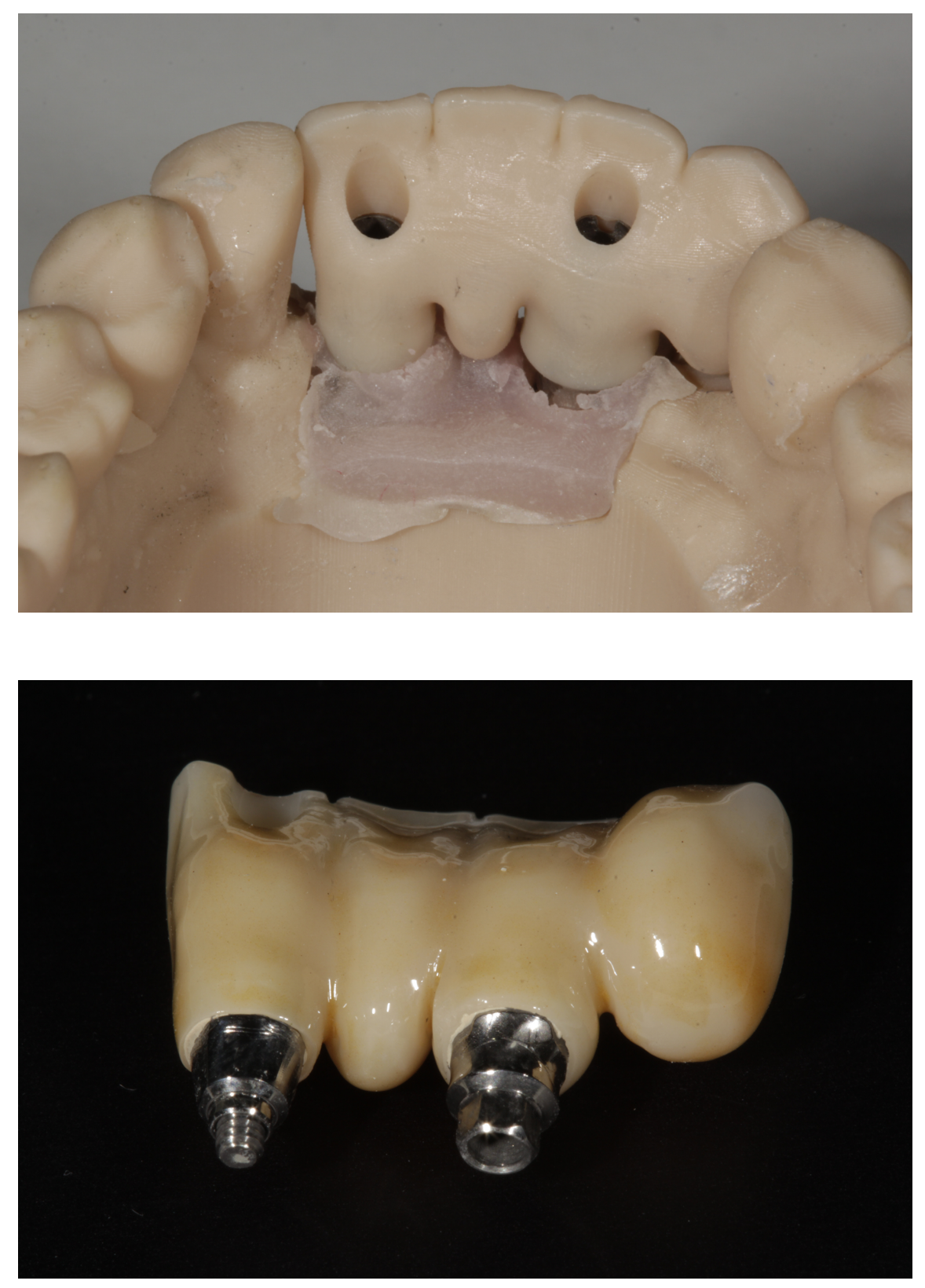

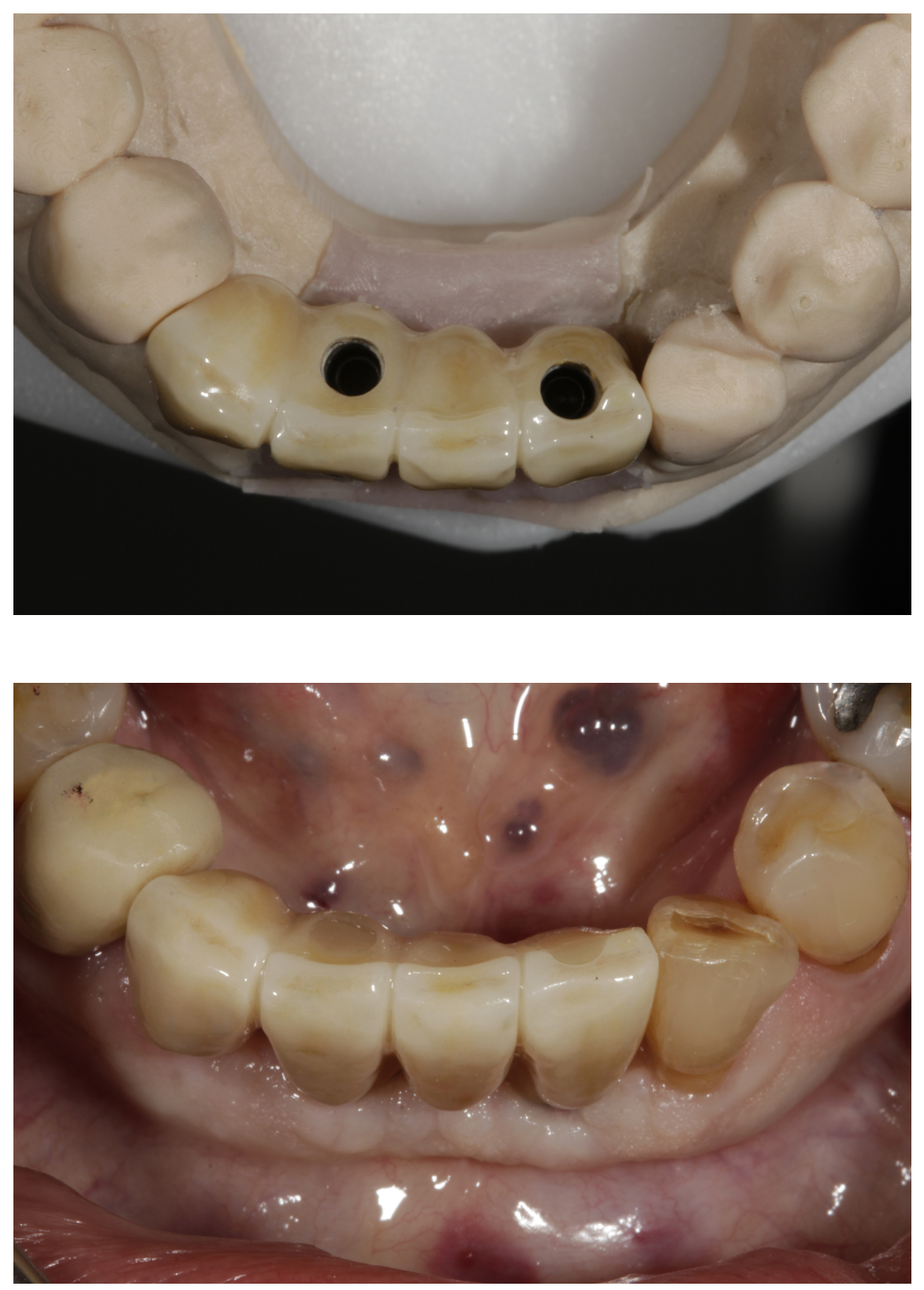